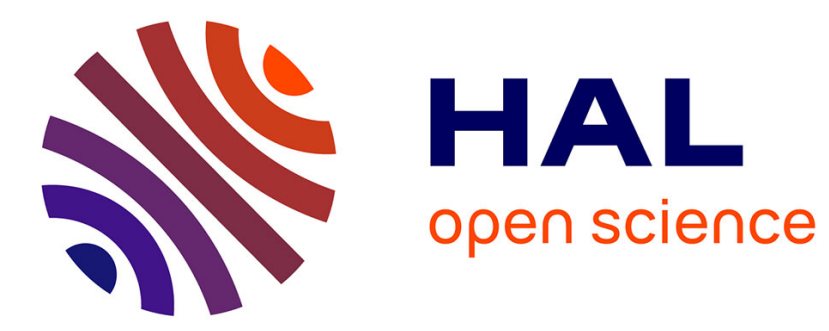

\title{
Decomposition of bivariate inequality indices by attributes
}

\author{
Ramses H. Abul Naga, Pierre-Yves Geoffard
}

\section{To cite this version:}

Ramses H. Abul Naga, Pierre-Yves Geoffard. Decomposition of bivariate inequality indices by attributes. 2005. halshs-00590715

\section{HAL Id: halshs-00590715 https://shs.hal.science/halshs-00590715}

Preprint submitted on 4 May 2011

HAL is a multi-disciplinary open access archive for the deposit and dissemination of scientific research documents, whether they are published or not. The documents may come from teaching and research institutions in France or abroad, or from public or private research centers.
L'archive ouverte pluridisciplinaire HAL, est destinée au dépôt et à la diffusion de documents scientifiques de niveau recherche, publiés ou non, émanant des établissements d'enseignement et de recherche français ou étrangers, des laboratoires publics ou privés. 
Decomposition of bivariate inequality indices

\author{
by attributes
}

Ramses H. Abul Naga

Pierre-Yves Geoffard

JEL Codes : D31, D63

Keywords : multidimensional inequality, relative indices, correlation increasing transfers, copulas. 


\title{
Decomposition of Bivariate Inequality Indices by Attributes
}

\author{
Ramses H. Abul Naga * and Pierre-Yves Geoffard †
}

June 17, 2005

\begin{abstract}
We provide, for the class of relative bidimensional inequality indices, a decomposition of inequality into two univariate AtkinsonKolm-Sen indices and a third statistic which depends on the joint distribution of resources.

Keywords: multidimensional inequality, relative indices, correlation increasing transfers, copulas.

JEL codes: $D_{31}, D_{63}$.
\end{abstract}

*Institute for Health Economics and Management (Université de Lausanne).

${ }^{\dagger}$ PSE (Paris Jourdan), Institute for Health Economics and Management (Université de Lausanne) and CEPR. 
When measuring inequality over two attributes, it may be of practical interest to express inequality as a function of the underlying level of inequality in each attribute. This note provides, for the class of relative bidimensional inequality indices, a decomposition of inequality into two univariate Atkinson-Kolm-Sen [AKS] indices ${ }^{1}$ and a third statistic which depends on the joint distribution of resources.

\section{Notation and definitions}

A Population consists of $n$ individuals. Individual $i$ has resources $x_{i} \doteq$ $\left(x_{i 1}, x_{i 2}\right)$, where $x_{i} \in R_{++}^{2}$. The joint distribution is a matrix $X \doteq\left[\begin{array}{l}x_{1} \\ \vdots \\ x_{n}\end{array}\right] \in$ $M^{n}$, the set of all $n \times 2$ matrices with strictly positive elements. Accordingly, the $j$ th column of $X, X_{j}$, gives a distribution (an $n \times 1$ vector) for the $j t h$ attribute in the population, and we also write $X=\left[\begin{array}{ll}X_{1} & X_{2}\end{array}\right]$.

A bidimensional inequality index is a real valued function $I(X): M^{n} \rightarrow$ $R_{+}$. Underlying $I$ is a social welfare function $W(X): M^{n} \rightarrow R$. We take $W($.$) to be the average of individual welfare levels:$

$A D D: W(X)=\frac{1}{n} \sum_{i} u\left(x_{i}\right)$ for any $X$ (additive separability).

$C O N: W($.$) is continuous in X$.

$P A R: W($.$) is strictly increasing in all its arguments (Pareto principle).$

$E Q U A L: W($.$) is a strictly quasi-concave function (preference for equal-$ ity).

$R S I N V$ : If $\Lambda$ is an $2 \times 2$ diagonal matrix with strictly positive elements, then $W\left(X^{A}\right)=W\left(X^{B}\right) \Longleftrightarrow W\left(X^{A} \Lambda\right)=W\left(X^{B} \Lambda\right)$, (ratio-scale invariance).

The above axioms on $W$ entail that $u($.$) is continuous, increasing, concave$ and ratio-scale invariant. In what follows, we shall refer to the set of axioms $A D D, C O N, P A R, E Q U A L$ and $R S I N V$ as the basic axioms.

Let $\mu \doteq\left(\mu_{1}, \mu_{2}\right)$ denote the vector of sample means and let $w^{o} \doteq W(X)$, be the level of welfare attained by $X$. Then, if $W($.$) satisfies the basic ax-$ ioms, we may define a scalar $\theta(X)$ in the unit interval, such that $u(\theta \mu)=$ $\frac{1}{n} \sum_{i} u\left(x_{i}\right)=w^{o}$. Starting from a distribution $X$, a fraction $\theta$ of the sumtotal of each attribute would lead to the same level of welfare as $X$, provided each attribute were equally distributed in the population. Therefore, $\theta$ is a

\footnotetext{
${ }^{1}$ See Atkinson (1970), Sen (1973), Kolm (1977) and Tsui (1995).
} 
measure of equality in $X$, and

$$
I(X)=1-\theta(X)
$$

is the corresponding relative inequality index (Kolm, 1977). Likewise, below we shall define equality indices $\theta_{j}$ for each separate attribute. Our aim is to decompose $\theta$ as a function of statistics $\theta_{1}, \theta_{2}$, pertaining to the marginal distributions and a term $\kappa$ which depends on the correlation between the two attributes.

Denote by $F_{12}$ the cumulative distribution function of $X$, and by $F_{j}$ the marginal distribution of the $j$-th attribute $X_{j}$. By Sklar's theorem (Sklar, 1959), there exists a copula function $c:[0,1] \times[0,1] \rightarrow[0,1]$ such that for any $\left(x_{1}, x_{2}\right), F_{12}\left(x_{1}, x_{2}\right)=c\left[F_{1}\left(x_{1}\right), F_{2}\left(x_{2}\right)\right]$. The copula captures the association between the two variables, and the joint distribution $F_{12}$ may be identified with its two marginal distributions and its copula function.

In general terms, we shall say that $\theta(X)$ is decomposable into two indices $\theta_{1}, \theta_{2}$, and a measure of association $\kappa$ if there exist four $R \rightarrow R$ functions $h(),. g^{1}(),. g^{2}($.$) , and \chi($.$) , where h, g^{1}$, and $g^{2}$ are monotonically increasing, such that:

$$
h\left[\theta\left(F_{12}\right)\right]=g^{1}\left[\theta_{1}\left(F_{1}\right)\right]+g^{2}\left[\theta_{2}\left(F_{2}\right)\right]+\chi\left[\kappa\left(c\left(F_{1}, F_{2}\right)\right)\right]
$$

where $\kappa($.$) measures the association between attributes via the copula func-$ tion.

Note that there are different ways to define equality in a given dimension, in particular with regard to whether the distribution over the other attribute has been equalized. Two polar cases may thus be defined. First, consider a situation where only $x_{2}$ is redistributed in the population. Then, we may define a scalar $\rho_{2}(X)$ such that $\frac{1}{n} \sum_{i} u\left(x_{i 1}, \rho_{2} \mu_{2}\right)=w^{o}$. Hence, $\rho_{2}$ is a measure of equality in $X_{2}$ holding the distribution $X_{1}$ constant. Next, there exists a scalar $\gamma_{1}(X)$, such that $u\left(\gamma_{1} \mu_{1}, \rho_{2} \mu_{2}\right)=\frac{1}{n} \sum_{i} u\left(x_{i 1}, \rho_{2} \mu_{2}\right)$. The scalar $\gamma_{1}$ is the fraction of $\mu_{1}$ that, if equally distributed in the population, would attain the same level of welfare as $X$, conditional on everyone receiving an endowment $\rho_{2} \mu_{2}$ of $x_{2}$. Both $\gamma_{1}$ and $\rho_{1}$ provide measures of equality in the first attribute, and we derive below inequalities relating $\gamma_{j}$ and $\rho_{j}$.

When $u($.$) is additively separable in x_{1}$ and $x_{2}$ we show that $\gamma_{j}=\rho_{j}$, and $\theta$ may be decomposed in terms of $\gamma_{1}$ and $\gamma_{2}$. In the more general context where $u($.$) is not separable across attributes, we show that \theta$ may be decomposed in terms of $\gamma_{1}, \gamma_{2}$, and a third index $\kappa$ that captures the correlation between 
the two attributes. Finally, we generalize our decomposition in the context of $p>2$ attributes.

\section{Inequality and the correlation between at- tributes}

It may certainly be advocated that changes in the joint distribution which increase the correlation between attributes for given marginal distributions, ought to modify inequality. Formally, let $x_{l}$ and $x_{m}$ denote any two rows of $X$, and define $y_{i} \doteq x_{i}$ for all $i \neq l, m$ and $y_{l j} \doteq \max \left\{x_{l j} ; x_{m j}\right\}, y_{m j} \doteq$ $\min \left\{x_{l j} ; x_{m j}\right\} j=1,2$; following Tsui (1999), we shall state that $Y$ is a correlation increasing transformation $(C I T)$ of $X$. Given that a $C I T$ does not affect the marginal distributions, it amounts to a transfer which increases the rank correlation between attributes (i.e. Kendall's Tau). The next axiom formalizes the argument $W(Y) \leq W(X)$ :

$C I T A V$ : For any $X$ and $Y$, if $Y$ is a CIT of $X$, then $W(Y) \leq W(X)$, (aversion to correlation increasing transformations).

To provide some intuition for the results to follow, suppose $x_{1}$ is income and $x_{2}$ is health. With $u_{12}<0$, the marginal utility of income is higher for the unhealthy. Therefore, a redistribution of income from a person in good health to a less healthy one may increase welfare, even if the latter is initially wealthier. The following lemma (Atkinson and Bourguignon, 1982) formalizes this argument.

Lemma Assume $W($.$) satisfies the basic axioms. If in addition W$ has an aversion (preference) for CITs, then $u_{12} \leq 0\left(u_{12} \geq 0\right)$.

If $W($.$) satisfies the basic axioms then u($.$) is one of three forms (Aczél,$ 1988; Tsui, 1995):

$$
\begin{aligned}
& u\left(x_{i 1}, x_{i 2}\right) \doteq x_{i 1}^{\alpha} x_{i 2}^{\beta} \quad \alpha, \beta>0, \quad \alpha+\beta \leq 1 \\
& u\left(x_{i 1}, x_{i 2}\right) \doteq-x_{i 1}^{\alpha} x_{i 2}^{\beta} \quad \alpha, \beta<0 \\
& u\left(x_{i 1}, x_{i 2}\right) \doteq \alpha \ln x_{i 1}+\beta \ln x_{i 2} \quad \alpha, \beta>0
\end{aligned}
$$

The first of these forms has $u_{12}>0$, i.e. $W($.$) is increasing in CITs. For the$ second form $u_{12}<0$, while for the latter $u_{12}=0$, i.e. $W($.$) is insensitive to$ CITs. We begin with this simpler case. 
Under (5), $u\left(x_{1}, x_{2}\right)$ is additively separable in $x_{1}$ and $x_{2}$; accordingly,

$$
w^{o}=\alpha \ln \theta \mu_{1}+\beta \ln \theta \mu_{2}=\alpha \ln \gamma_{1} \mu_{1}+\beta \ln \gamma_{2} \mu_{2}
$$

Upon defining $\omega \doteq \alpha /(\alpha+\beta)$, we obtain the elasticity decomposition

$$
\ln \theta(X)=\omega \ln \gamma_{1}\left(X_{1}\right)+(1-\omega) \ln \gamma_{2}\left(X_{2}\right) \quad \text { when } \quad u_{12}=0
$$

so that a $1 \%$ increase in equality of the distribution $X_{1}$ results in a $\omega \%$ increase in the overall level of equality $\theta$. Notice also here that $\rho_{j}=\gamma_{j}=$ $\exp \left[\frac{1}{n} \sum_{i} \ln x_{i j}-\ln \mu_{j}\right]$.

For the general case $u_{12} \neq 0$, it is usually not possible to modify the distribution $X_{1}$ while holding $\gamma_{2}$ constant. The elasticity decomposition is given in the following proposition.

Proposition 1: Assume $W($.$) satisfies the basic axioms. Then$

$$
\ln \theta(X)=\omega \ln \gamma_{1}\left(X_{1}\right)+(1-\omega) \ln \gamma_{2}\left(X_{2}\right)+\frac{1}{(\alpha+\beta)} \ln \kappa(X)
$$

where $\gamma_{j}$ is an $A K S$ index for $X_{j}$ and $\kappa$ is given by

$$
\kappa \doteq \frac{n \sum_{i} x_{i 1}^{\alpha} x_{i 2}^{\beta}}{\sum_{i} x_{i 1}^{\alpha} \sum_{i} x_{i 2}^{\beta}}
$$

Proof: Consider first (3). From the definition of $\theta$, it follows that $w^{o}=$ $\left(\theta \mu_{1}\right)^{\alpha}\left(\theta \mu_{2}\right)^{\beta}=\left(\gamma_{1} \mu_{1}\right)^{\alpha}\left(\rho_{2} \mu_{2}\right)^{\beta}$. Therefore, $(\alpha+\beta) \ln \theta=\alpha \ln \gamma_{1}+\beta \ln \rho_{2}$. In other terms,

$$
\ln \theta=\omega \ln \gamma_{1}+(1-\omega) \ln \gamma_{2}+(1-\omega) \ln \left(\rho_{2} / \gamma_{2}\right)
$$

Likewise, $w^{o}=\left(\rho_{1} \mu_{1}\right)^{\alpha}\left(\gamma_{2} \mu_{2}\right)^{\beta}=\frac{1}{n} \sum_{i}\left(\rho_{1} \mu_{1}\right)^{\alpha} x_{i 2}^{\beta}$. Hence,

$$
\gamma_{2}=\frac{1}{\mu_{2}}\left(\frac{1}{n} \sum_{i} x_{i 2}^{\beta}\right)^{1 / \beta}
$$

Since $w^{o}=\frac{1}{n} \sum_{i} x_{i 1}^{\alpha} x_{i 2}^{\beta}=\frac{1}{n} \sum_{i} x_{i 1}^{\alpha}\left(\rho_{2} \mu_{2}\right)^{\beta}$, we obtain

$$
\rho_{2}=\frac{1}{\mu_{2}}\left(\frac{\sum_{i} x_{i 1}^{\alpha} x_{i 2}^{\beta}}{\sum_{i} x_{i 1}^{\alpha}}\right)^{1 / \beta}
$$


Together, (11) and (12) give $(1-\omega) \ln \left(\rho_{2} / \gamma_{2}\right)=(\ln \kappa) /(\alpha+\beta)$, which completes the proof for (3). For (4), it is readily verified that $\gamma_{2}$ and $\rho_{2}$ are also given as in (11) and (12) above.

If $Y$ is a CIT of $X$, it is the case that $F_{j}\left(Y_{j}\right)=F_{j}\left(X_{j}\right)$. Hence, $\gamma_{j}(Y)=$ $\gamma_{j}(X)$. On the basis of $(8)$, we therefore obtain

$$
\ln \theta(Y)-\ln \theta(X)=\frac{1}{(\alpha+\beta)}[\ln \kappa(Y)-\ln \kappa(X)]
$$

Since $\kappa$ is a measure of association between the two attributes, it is desirable that this function be rising in correlation increasing transformations. The next proposition formalizes this argument.

Proposition 2: If $Y$ is a CIT of $X$, then $\kappa(Y) \geq \kappa(X)$.

Proof: For $j=1,2$ let $\underline{x}_{j}<\bar{x}_{j}$. If $Y$ is a CIT of $X$, we may take $x_{l} \doteq$ $\left(\bar{x}_{1}, \underline{x}_{2}\right), x_{m} \doteq\left(\underline{x}_{1}, \bar{x}_{2}\right), y_{l} \doteq\left(\bar{x}_{1}, \bar{x}_{2}\right), y_{m} \doteq\left(\underline{x}_{1}, \underline{x}_{2}\right)$ and $y_{i} \doteq x_{i}$ for all $i \neq l, m$. Since the marginal distributions are unaffected by CITs, it follows from (9) that $\kappa(Y) \geq \kappa(X)$ when the difference $\Delta \doteq \bar{x}_{1}^{\alpha} \bar{x}_{2}^{\beta}+\underline{x}_{1}^{\alpha} \underline{x}_{2}^{\beta}-\bar{x}_{1}^{\alpha} \underline{x}_{2}^{\beta}-\underline{x}_{1}^{\alpha} \bar{x}_{2}^{\beta}$ is positive. Since $\Delta \doteq\left(\bar{x}_{1}^{\alpha}-\underline{x}_{1}^{\alpha}\right)\left(\bar{x}_{2}^{\beta}-\underline{x}_{2}^{\beta}\right)$, we have that $\Delta \geq 0$ when $\alpha$ and $\beta$ are of the same sign.

Returning to (13) we note that when the social welfare function is averse to correlation increasing transformations $(\alpha, \beta<0)$, a $C I T$ results in a reduction in $\theta$, that is, an increase in inequality. In other terms, the change in inequality that results from a $C I T$ is captured via $\kappa$.

\section{Some inequalities}

Recall that $\rho_{2}$ is a measure of equality in $X_{2}$ holding the distribution $X_{1}$ constant, whereas $\gamma_{2}$ measures the level of equality in $X_{2}$ having equalized the distribution $X_{1}$. Consider (4) where $W($.$) is averse to CITs. Then, if the$ correlation between $x_{1}^{\alpha}$ and $x_{2}^{\beta}$ is positive, we expect the level of equality in $X_{2}$ to be greater after equalizing the distribution $X_{1}$ than before, i.e. that $\gamma_{2} \geq \rho_{2}$. We have:

Proposition 3: In the context of $(3), \gamma_{j} \leq \rho_{j}$ iff the correlation between $x_{1}^{\alpha}$ and $x_{2}^{\beta}$ is positive. For (4), $\gamma_{j} \geq \rho_{j}$ iff the correlation between $x_{1}^{\alpha}$ and $x_{2}^{\beta}$ is positive. For (5), $\gamma_{j}=\rho_{j}$. 
Recall that $\kappa$ is a measure of association between $x_{1}$ and $x_{2}$. It is greater than (less than) unity when $x_{1}^{\alpha}$ and $x_{2}^{\beta}$ are positively (negatively) correlated. The index $\kappa$ is a simple transform of the ratio of $\rho_{j}$ to $\gamma_{j}$ :

$$
\kappa=\left(\frac{\rho_{2}}{\gamma_{2}}\right)^{\beta}=\left(\frac{\rho_{1}}{\gamma_{1}}\right)^{\alpha}
$$

In the context of (3), $\alpha, \beta>0$ while in (4) $\alpha, \beta<0$, from which the results of the proposition follow.

\section{Generalization}

A final point we turn to is the decomposition of inequality in the general context of $p>2$ attributes. Define $x_{i} \doteq\left(x_{i 1}, \ldots, x_{i p}\right)$. For $u\left(x_{i}\right)$ to satisfy the basic axioms it must take one of the following forms: $u\left(x_{i}\right) \doteq x_{i 1}^{\alpha_{1}} \ldots \ldots$ $x_{i p}^{\alpha_{p}}, \alpha_{j}>0$ for all $j$, and $\sum_{j} \alpha_{j} \leq 1 ; u\left(x_{i}\right) \doteq-x_{i 1}^{\alpha_{1}} \cdots \cdots x_{i p}^{\alpha_{p}}, \alpha_{j}<0$ for all $j$ and finally, $u\left(x_{i}\right) \doteq \sum_{j} \alpha_{j} \ln x_{i j}$, where $\alpha_{j}>0$ for all $j$. Propositions 1 and 2 generalize to higher dimensions. The decomposition of inequality in the context of $p$ attributes is given by

$$
\begin{aligned}
\left(\sum_{j} \alpha_{j}\right) \ln \theta & =\sum_{j} \alpha_{j} \ln \gamma_{j}+\ln \kappa \\
\kappa & \doteq \frac{n \sum_{i} x_{i 1}^{\alpha_{1}} \cdots x_{i p}^{\alpha_{p}}}{\sum_{i} x_{i 1}^{\alpha_{1}} \cdots \sum_{i} x_{i p}^{\alpha_{p}}}
\end{aligned}
$$

As before, $\gamma_{j}$ denotes an AKS equality index for the distribution $X_{j}$, whereas now $\kappa$ is a function of the product moment between the variables $x_{1}^{\alpha_{1}}, \ldots, x_{p}^{\alpha_{p}}$ thus generalizing the earlier bivariate measure of association (9).

\section{References}

Aczél J. (1988): A Short Course on Functional Equations, Dordrecht, Reidel.

Atkinson A. (1970): "On the Measurement of Inequality", Journal of Economic Theory 2, 244-263.

Atkinson A. and F. Bourguignon (1982): "The Comparison of MultiDimensioned Distributions of Economic Status", Review of Economic Studies 49, 183-201. 
Kolm S. (1977): "Multidimensional Egalitarianisms", Quarterly Journal of Economics 91, 1-13.

Sen A. (1973): On Economic Inequality, Oxford, Clarendon Press.

Sklar A. (1959): "Fonctions de Répartition à $n$ dimensions et leurs marges", Publications de l'Institut de Statistique de l'Université de Paris, 8, 229-231.

Tsui K-Y (1995): "Multidimensional Generalizations of the Relative and Absolute Inequality Indices: The Atkinson-Kolm-Sen Approach", Journal of Economic Theory 67, 251-265.

Tsui K-Y (1999): "Multidimensional Inequality and Multidimensional Entropy Measures: An Axiomatic Derivation", Social Choice and Welfare $16,145-157$. 